MS-TP-01-12

Bicocca-FT-01-21

\title{
Quenched twisted mass QCD at small quark masses and in large volume*
}

\author{
M. Della Morte ${ }^{\mathrm{a}}$, R. Frezzotti ${ }^{\mathrm{a}}$ and J. Heitger ${ }^{\mathrm{b}}$ \\ a Università di Milano-Bicocca, Dipartimento di Fisica, Piazza della Scienza 3, I-20126 Milano, Italy \\ bWWU Münster, Institut für Theoretische Physik, Wilhelm-Klemm-Str. 9, D-48149 Münster, Germany
}

As a test of quenched lattice twisted mass QCD, we compute the non-perturbatively $\mathrm{O}(a)$ improved pseudoscalar and vector meson masses and the pseudoscalar decay constant down to $M_{\mathrm{PS}} / M_{\mathrm{V}}=0.467(13)$ at $\beta=6$ in large volume. We check the absence of exceptional configurations and - by further data at $\beta=6.2$ - the size of scaling violations. The CPU time cost for reaching a given accuracy is close to that with ordinary Wilson quarks at $M_{\mathrm{PS}} / M_{\mathrm{V}} \simeq 0.6$ and grows smoothly as $M_{\mathrm{PS}} / M_{\mathrm{V}}$ decreases.

\section{INTRODUCTION}

The lattice twisted mass QCD (tmQCD) action [1] in infinite space-time volume reads

$$
\begin{gathered}
S_{\mathrm{W}}[U, \bar{\psi}, \psi]=S_{\mathrm{g}}+a^{4} \sum_{x} \bar{\psi}_{x}\left[D_{\mathrm{tm}} \psi\right]_{x}, \\
D_{\mathrm{tm}}=\gamma \widetilde{\nabla}-\frac{a}{2} \nabla^{*} \nabla+m_{0}+i \mu_{\mathrm{q}} \gamma_{5} \tau^{3},
\end{gathered}
$$

where $\widetilde{\nabla}=\left(\nabla+\nabla^{\star}\right) / 2, \psi$ is a flavour quark doublet and the matrix $\tau^{3}$ acts in the flavour space. If $m_{0} \equiv m_{\mathrm{c}}$, the quark mass is entirely given by the multiplicatively renormalized parameter $\mu_{\mathrm{q}}$.

Lattice tmQCD is a regularization of QCD with $N_{\mathrm{f}}=2$ mass degenerate quarks [1] where an action term that is proportional to $\mu_{\mathrm{q}}$ and is not aligned in flavour chiral space with the Wilson term contributes to the soft breaking of the chiral symmetry. As a consequence, the lowest eigenvalue of the Hermitean square of the Dirac matrix on any gauge background is bounded from below - in lattice units - by $\left(a \mu_{\mathrm{q}}\right)^{2}$. The problem of the exceptional configurations in quenched and partially quenched QCD is thus solved as long as the quark mass is not vanishingly small.

\footnotetext{
${ }^{*}$ Based on a poster presented by R. Frezzotti at the XIX International Symposium on Lattice Field Theory "Lattice 2001", August 19-24, 2001, Berlin, Germany.
}

One can think of the two mass-degenerate quark flavours as the $u$ and $d$ quarks in a chirally twisted basis, with the obvious consequences on the physical meaning of the correlation functions [1]. Heavier flavours of Wilson quarks can still be added e.g. in the usual way, while the neglected tiny mass difference between $u$ and $d$ quarks can safely be taken into account by means of chiral perturbation theory. Moreover, lattice tmQCD can also be formulated for a doublet of non-degenerate quarks, whilst retaining the protection against exceptionals on all gauge backgrounds [4].

\section{OBSERVABLES AND SIMULATIONS}

In this study we restrict attention to the pseudoscalar and vector meson masses, $M_{\mathrm{PS}}$ and $M_{\mathrm{V}}$, the pseudoscalar decay constant, $F_{\mathrm{PS}}$, and the renormalized quark masses, $\mu_{\mathrm{R}}$ and $m_{\mathrm{R}}$, which are defined according to eq. (3.3) and eq. (3.20) of Ref. [3]. It is convenient for our discussion to define polar quark mass "coordinates" via

$$
\tan \alpha=\mu_{\mathrm{R}} / m_{\mathrm{R}}, \quad M_{\mathrm{R}}=\left(\mu_{\mathrm{R}}^{2}+m_{\mathrm{R}}^{2}\right)^{1 / 2} .
$$

We work in the quenched approximation considering systems of physical size $L^{3} T$ at $\beta=6$ and 


\begin{tabular}{ccccccccc}
\hline Set & $\beta$ & $\kappa, \mu_{\mathrm{q}}$ & $L / a, T / L$ & \# meas. & $M_{\mathrm{R}} r_{0}$ & $M_{\mathrm{PS}} r_{0}$ & $F_{\mathrm{PS}} r_{0}$ & $M_{\mathrm{V}} r_{0}$ \\
\hline A1 & 6 & $0.135196,0.0266$ & 16,2 & 650 & $0.2729(15)$ & $1.711(7)$ & $0.455(5)$ & $2.662(40)$ \\
A1 & 6 & $0.135196,0.0266$ & 16,3 & 650 & $0.2729(15)$ & $1.714(6)$ & $0.455(6)$ & $2.656(42)$ \\
A2 & 6.2 & $0.135814,0.0180$ & 24,2 & 535 & $0.2558(16)$ & $1.623(8)$ & $0.456(5)$ & $2.557(32)$ \\
B1L & 6 & $0.135208,0.0190$ & 24,2 & 260 & $0.1949(11)$ & $1.452(6)$ & $0.432(6)$ & $2.517(35)$ \\
B1 & 6 & $0.135208,0.0190$ & 16,2 & 535 & $0.1949(11)$ & $1.455(8)$ & $0.428(5)$ & $2.513(47)$ \\
B2 & 6.2 & $0.135814,0.0138$ & 24,2 & 300 & $0.1962(12)$ & $1.420(9)$ & $0.436(7)$ & $2.462(41)$ \\
C & 6 & $0.135208,0.0117$ & 24,2 & 260 & $0.1205(7)$ & $1.160(6)$ & $0.401(6)$ & $2.485(59)$ \\
\hline
\end{tabular}

Table 1

$\beta=6.2$, with $L$ such that $M_{\mathrm{PS}} L \geq 4.5$ to suppress finite volume effects. Following closely Ref. [3], we adopt Schrödinger functional (SF) boundary conditions for tmQCD and compute the SF correlators

$f_{\mathrm{A}}^{11}\left(x_{0}\right), \quad f_{\mathrm{P}}^{11}\left(x_{0}\right), \quad f_{\mathrm{V}}^{12}\left(x_{0}\right), \quad f_{1}^{11}$,

$k_{\mathrm{V}}^{11}\left(x_{0}\right), \quad k_{\mathrm{T}}^{11}\left(x_{0}\right), \quad k_{\mathrm{A}}^{12}\left(x_{0}\right)$

as a function of the Euclidean time $x_{0}$, for $L$ ranging from 1.5 to $2.2 \mathrm{fm}$, depending on the quark mass values, and $T / L=2$ or 3 . We hence construct the renormalized and $\mathrm{O}(a)$ improved $\mathrm{SF}$ correlators $\left[f_{\mathrm{A}^{\prime}}^{11}\right]_{\mathrm{R}}\left(x_{0}\right),\left[f_{\mathrm{P}^{\prime}}^{11}\right]_{\mathrm{R}}\left(x_{0}\right),\left[k_{\mathrm{V}^{\prime}}^{11}\right]_{\mathrm{R}}\left(x_{0}\right)$ and $\left[k_{T^{\prime}}^{11}\right]_{\mathrm{R}}\left(x_{0}\right)$, which are specified in eq. (3.11) of Ref. [3] with $\alpha$ given by eq. (2).

In the limit of large $x_{0}$ and large $T-x_{0}$ and up to cutoff effects, the correlators $\left[f_{\mathrm{A}^{\prime}}^{11}\left(x_{0}\right)\right]_{\mathrm{R}}$ and $\left[f_{\mathrm{P}^{\prime}}^{11}\left(x_{0}\right)\right]_{\mathrm{R}}$, which correspond to the insertion of the operators $\left(A_{\mathrm{R}}^{\prime}\right)_{0}^{1}$ and $\left(P_{\mathrm{R}}^{\prime}\right)^{1}$, see eq. (3.10) of Ref. [3], are expected to be dominated by the lowest isotriplet pseudoscalar state. In the same limit the correlators $\left[k_{\mathrm{V}^{\prime}}^{11}\left(x_{0}\right)\right]_{\mathrm{R}}$ and $\left[k_{T^{\prime}}^{11}\left(x_{0}\right)\right]_{\mathrm{R}}$, which correspond to the insertion of the operators $\left(V_{\mathrm{R}}^{\prime}\right)_{k}^{1}$ and $\left(T_{\mathrm{R}}^{\prime}\right)_{k 0}^{1}$, see eq. (3.10) of Ref. [3], are dominated by the lowest isotriplet vector state.

In our simulations we employ the nonperturbatively improved version of the action (1), with $c_{\mathrm{sw}}$ taken from Ref. [5]. An overview of our simulation parameters, statistics and preliminary results is given in Table 1, while $\alpha=\pi / 2+\mathrm{O}(a)$.

Our most critical simulation (set $\mathrm{C}$ ) required $\sim 230$ GFlops $\times$ day by employing a CGNE solver for the SSOR-preconditioned Dirac matrix $D_{\mathrm{tm}}$. For this (and similar) choice(s) of parameters the BiCG solver often does not converge, while a CGNE solver works fine with small fluctuations in the number of iterations.

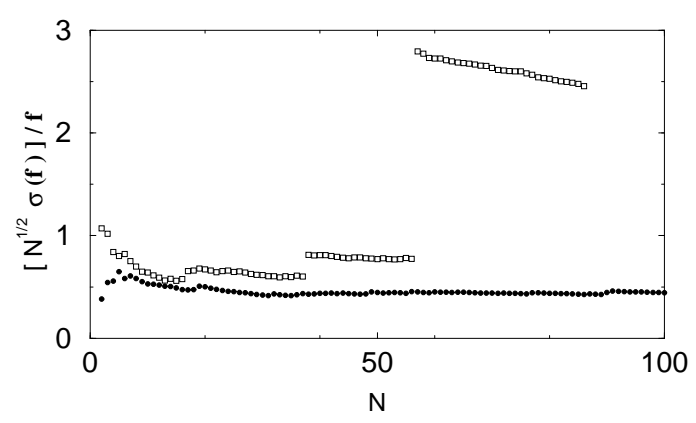

Figure 1. The square root of the relative a priori variance of $f=f_{\mathrm{P}}^{11}(24 a)$ versus the number of measurements $N$ for the simulation $\mathrm{C}$ (filled circles) and a simulation with the same values of $\beta$ and $M_{\mathrm{R}}$, but $\mu_{\mathrm{q}}=0$ (open squares).

\section{RESULTS}

We find that lattice tmQCD allows, as expected, to safely work in a region of parameters which would be inaccessible with ordinary Wilson quarks: see e.g. Fig. 1. For a given number of independent measurements, the statistical errors on $M_{\mathrm{PS}}$ and $M_{\mathrm{V}}$ are comparable, up to a factor of one to three, to those found e.g. with domain wall quarks [6]. The CPU time effort, e.g. for the data sets A1, A1' and A2, is in line with the computational cost for ordinary Wilson quarks.

Concerning the extraction of hadron masses and matrix elements, we recall that the lattice action (11) enjoys reduced parity and isospin symmetries 1]: it just preserves the third isospin generator and parity combined with a flavour exchange. At finite $a$ the correlators may hence receive contributions of order $a \mu_{\mathrm{q}}$ from the allowed states with different parity and isospin. While deferring details to Ref. 8], we show in Fig. 2 an example of effective masses extracted from SF correlators: 


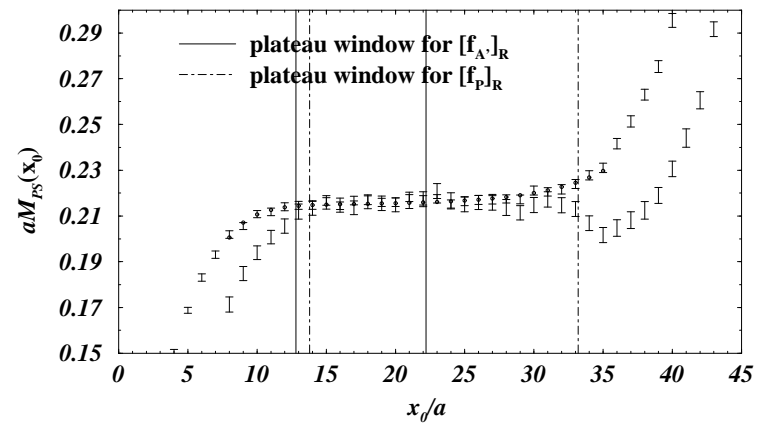

Figure 2. Pseudoscalar effective masses extracted from the correlators $\left[f_{\mathrm{A}^{\prime}}^{11}\right]_{\mathrm{R}}$ and $\left[f_{\mathrm{P}^{\prime}}^{11}\right]_{\mathrm{R}}$ for data set C. The circles denote our fit to $\left[f_{\mathrm{A}^{\prime}}^{11}\right]_{\mathrm{R}}$, which accounts for $\mathrm{O}\left(a \mu_{\mathrm{q}}\right)$ contributions from states with twisted parity and isospin at large $x_{0}$.

the correlator $\left[f_{\mathrm{A}^{\prime}}^{11}\right]_{\mathrm{R}}\left(x_{0}\right)$ receives contributions of order $a \mu_{\mathrm{q}}$ that are peculiar to our SF setup and would be absent at $\alpha=0$. Analogous effects in $\left[f_{\mathrm{P}^{\prime}}^{11}\right]_{\mathrm{R}}\left(x_{0}\right)$ are expected to be very small [8].

As detailed in Ref. [3], we expect the relations among our observables and the renormalized parameters $r_{0}$ and $M_{\mathrm{R}}$ to be $\mathrm{O}(a)$ improved. In particular, when working at $\alpha=\pi / 2+\mathrm{O}(a)$, an $\mathrm{O}(a)$ improved estimate of $F_{\mathrm{PS}}$ is obtained in terms of $M_{\mathrm{PS}}$ and the matrix element of e.g. the operator $2 M_{\mathrm{R}}\left(P_{\mathrm{R}}^{\prime}\right)^{1}$ between the charged pion and the vacuum [9]. This approach to $F_{\mathrm{PS}}$, which is also valid in unquenched lattice tmQCD, requires to know no other counterterms than $Z_{\mathrm{g}}, m_{\mathrm{c}}$ and $c_{\mathrm{sw}}$.

In order to check for the residual scaling violations, we produced data at $\beta=6.2$ (sets A2 and B2), while keeping $\alpha, M_{\mathrm{R}}$ and $r_{0}$ fixed. More precisely, the ratio $m_{\mathrm{R}} / M_{\mathrm{R}}$ was tuned 2 to the value $0.000(1)$ for $M_{\mathrm{R}} r_{0}=0.195(1)$ and $-0.015(1)$ for $M_{\mathrm{R}} r_{0}=0.273(2)$. For the simulation $\mathrm{C}$ we found $m_{\mathrm{R}} / M_{\mathrm{R}}=0.083(5)$. We also reanalysed the data of Ref. [7], which were produced at $\beta=6,6.1,6.2,6.45$, by imposing precisely the same renormalization conditions as in this study of tmQCD. We then performed a continuum extrapolation, assuming a purely quadratic dependence on $\left(a / r_{0}\right)^{2}$ and discarding the data at $\beta=6$. However, owing to the use of the one-loop estimate of $b_{\mathrm{A}}$, the resulting estimate of $F_{\mathrm{PS}}$ is

\footnotetext{
${ }^{2}$ The small mismatch in $M_{\mathrm{R}} r_{0}$ for the set A2 was corrected by employing estimates of the dependence of our observables on $M_{\mathrm{R}} r_{0}$.
}
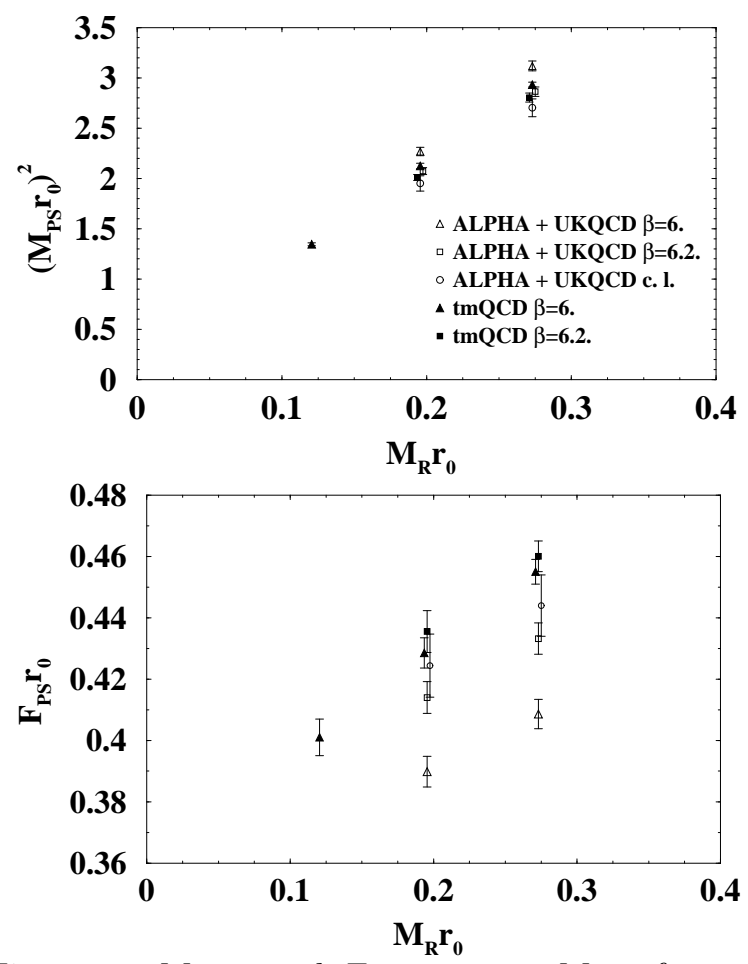

Figure 3. $\quad M_{\mathrm{PS}} r_{0}$ and $F_{\mathrm{PS}} r_{0}$ versus $M_{\mathrm{R}} r_{0}$ from tmQCD and reanalysis of the data of Ref. [7], including a continuum extrapolation (c. l.).

not fully $\mathrm{O}(a)$ improved. The outcome of this exercise is compared with the results from tmQCD in Fig. 3. We omit the case of $M_{\mathrm{V}} r_{0}$, as cutoff effects are hardly visible within statistical errors.

\section{CONCLUSIONS}

Lattice tmQCD solves the problem of exceptional configurations and allows for rather precise results with a moderate computational effort and tiny scaling violations, at least for the observables of this study. The framework is suitable for extension beyond the quenched approximation.

This work is part of the ALPHA Collaboration research programme.

\section{REFERENCES}

1. R. Frezzotti, P.A. Grassi, S. Sint and P. Weisz, Nucl. Phys. B (Proc. Suppl.) 83-84 (2000) 941; JHEP 0108 (2001) 058.

2. R. Frezzotti, S. Sint and P. Weisz, JHEP 0107 (2001) 048. 
3. M. Della Morte, R. Frezzotti, J. Heitger and S. Sint, hep-lat/0108019.

4. R. Frezzotti, unpublished notes.

5. M. Lüscher et al., Nucl. Phys. B491 (1997) 323.

6. A. Ali Khan et al., hep-lat/0105020.

7. J. Garden, J. Heitger, R. Sommer and H. Wittig, Nucl. Phys. B571 (2000) 237.

8. M. Della Morte, R. Frezzotti and J. Heitger, work in preparation.

9. R. Frezzotti and S. Sint, these proceedings. 